\title{
Towards a Framework for Enabling Operations of Livestock Information Systems in Poor Connectivity Areas
}

\author{
Herbert Peter Wanga, Khamisi Kalegele \\ School of Computational and Communication Sciences and Engineering (CoCSE), Nelson Mandela African Institution of Science and \\ Technology (NM-AIST), Arusha, Tanzania
}

Email address:

wangah@nm-aist.ac.tz (H. P. Wanga), khamisi.kalegele@nm-aist.ac.tz (K. Kalegele)

\section{To cite this article:}

Herbert Peter Wanga, Khamisi Kalegele. Towards a Framework for Enabling Operations of Livestock Information Systems in Poor Connectivity Areas. American Journal of Software Engineering and Applications. Vol. 4, No. 3, 2015, pp. $42-49$.

doi: $10.11648 /$ j.ajsea.20150403.11

\begin{abstract}
Livestock farming is one of the major agricultural activities in the country that is contributing towards achieving development goals of the national Growth and Reduction of Poverty (NSGRP). Smallholder livestock keepers depend on the information from the livestock field officers for sound decision making. Mobile application based solutions, which are currently widely proposed to facilitate the process, fail to perform in poor connectivity areas. This study proposes a machine learning based framework which will enhance the performance of mobile application based solutions in poor connectivity areas. The study used primary data, and secondary data. The primary data were collected through surveys, questionnaires, interviews, and direct observations. Secondary data were collected through books, articles, journals, and Internet searching. Open Data Kit (ODK) tool was used to collect responses from the respondents, and their geographical positions. We used Google earth to have smallholder livestock keepers' distribution map. Results show that smallholder livestock keepers are geographically scattered and depend on the field livestock officers for exchange of information. Their means of communication are mainly face to face, and mobile phones. They do not use any Livestock Information System. The proposed framework will enable operations of Livestock Information System in poor connectivity area, where majority of smallholder livestock keepers live. This paper provides the requirements model necessary for designing and development of the machine learning-based application framework for enhancing performance of livestock mobile application systems, which will enable operations of livestock information systems in poor connectivity areas.
\end{abstract}

Keywords: Livestock, Information System, Machine Learning, Mobile Application, Technology, Smartphone

\section{Introduction}

This section introduces the study by providing the general overview. It covers the information system development, livestock in Tanzania, usefulness of machine learning (ML), and objective of the study.

\subsection{Information System Development}

Usually, information system development projects start with textual requirements descriptions and transform these into a conceptual schema based on a particular conceptual model (e.g., using the UML) [1].

Use case analysis is one particular technique that has shown value for specifying requirements for many different types of systems. Use case analysis has gained prominence due to its inclusion in the Unified Modeling Language (UML) and therefore has gained particular prominence in the construction of object oriented systems [2].

The UML supports a standard notation for use cases and actors. Participation in a particular use case by an actor is shown by a line between the actor and the use case. The notation allows for requirements to be abstracted and encapsulated in a structure that is logical and concise [2].

The process of requirements gathering and analysis has, according to [3] main objectives: To provide system developers with a better understanding of stakeholder needs, which are the professionals directly involved with the project from the end user to responsible for approving the project development; Define the limits of the system (system scope); provide a basis for planning the technical content of the 
stages of development; provide a basis for estimating cost and development time of the system; define a user interface for the system, focusing on the needs and goals of the users; Establish and maintain compliance with customers and other stakeholders about what the system should do.

\subsection{Livestock in Tanzania}

According to [4], Livestock farming is one of the major agricultural activities in the country that is contributing towards achieving development goals of the national Growth and Reduction of Poverty (NSGRP). The livestock industry contribution to the Agricultural Gross Domestic product is about $13 \%$, and contributed $4.0 \%$ of the National Gross Domestic product in 2009 compared to $4.7 \%$ in 2008 .

Despite of its contribution to the economy of the country, the livestock sector faces a number of challenges, including geographical dispersion of livestock stakeholders, inadequate number of livestock workers, and poor access of agricultural information.

\subsection{Usefulness of Machine Learning (ML)}

Machine learning is a field that is at the intersection of computer science, statistics, and applied mathematics. It is the study of algorithms that improve their performance based on experience. It is a branch of Artificial Intelligence which focuses on the construction and study of systems that can learn from data. It is a science of getting computers to act without being explicitly programmed. Machine Learning enables computers to learn and act based on their knowledge.

We use Machine Learning in our daily lives, sometimes without our knowledge: our email service uses machine learning to identify spam or malicious emails and removes them from our inbox, the search engines use machine learning to analyze and evaluate websites and answer our queries etc.

Employing machine learning techniques to livestock information systems in poor connectivity areas, allow the system to intelligently predict the user's demand, provide services accordingly and save the human users' attention cost significantly.

Their implicit predictive ability could be embedded in automatic processes such as expert systems, or they could be used directly for communication between human experts and for educational purposes [5].

One of the practical problems in applying machine learning is that it is hard to acquire a variety of learning tools and experiment with them in a uniform way. We describe a software workbench, called WEKA, which collects together a number of schemes and allows users to run them on realworld data sets and interpret and compare the results [5].

Most learning techniques that have actually been applied assume that the data are presented in a simple attribute-value format in which a record has a fixed number of constantvalued fields or properties [5].

Machine learning techniques will allow the android application to respond to the livestock keepers in poor connectivity area, where majority of them live. In addition, it will assist the application to make predictions.

\subsection{Objective of the Study}

The objective of the study is to improve farm-level decision making in poor connectivity areas using a machine learning based framework.

This study was conducted in the Arumeru district, Arusha region, which is found in the northern zone of Tanzania. Data were collected from fifteen wards, namely Poli, Sing'isi seela, Makiba, Maroroni, Mbuguni, Akheri, Kikatiti, Kikwe, King'ori, Maji ya chai, Nkoanrua, Nkoaranga, Nkoarisambu, Songoro, and Usa river.

The study identified four key user groups of the system, namely smallholder livestock keepers, field livestock officers, district livestock officers, and livestock researchers. Generally, field livestock officers, district livestock officers, and livestock researchers are called Livestock experts.

This paper presents users and system requirements as a result of data analysis obtained from questionnaires, interviews, direct observations, and document reviews.

It was observed that, majority of smallholder livestock keepers depend on the information delivered from livestock field officers.

The remaining part of this paper is organized as follows. Section 2 briefly describes literature review, Next, Section 3 presents methodology, and Whereas Section 4 covers results and discussion. Section 5 concludes the paper, and explains the future work.

\subsection{Our Contribution}

Our main contribution here is the intelligent livestock Information System, which is a novel idea in the field of mobile livestock information systems. This system will work in both internet and poor internet connectivity areas.

The study paves a way for a machine learning based framework which will enhance the performance of mobile application based solutions in poor connectivity areas.

\section{Literature Review}

The study went through the related works. It covers a number of aspects, which are requirements definition, importance of requirements gathering, attributes of good requirements, requirements analysis, and studies of livestock information systems.

\subsection{Requirements Definition}

Reference [6] suggests that requirement is what the system must be able to do. It is a process or improvement, which stakeholders want to realize through a system. Requirements are for users, stakeholder customers, service providers, development team, testers/ validation.

Requirements definition is a careful assessment of the needs that a system is to fulfill...must say why a system is needed, based on current and foreseen conditions, which may 
be internal operations or an external market...must say what system features will serve and satisfy this context...must also say how the system is to be constructed [7].

A requirement is a description of a system feature, capability, or constraint. Requirements generally focus on what a system should do, rather than how it should do it [8].

Classes of Requirements are functional, and nonfunctional (constraints)

\subsection{Importance of Requirements Gathering}

According to [9], user requirements play a fundamental role in restricting the area of interest for data analysis and in choosing facts, dimensions, and measures for system design and implementation.

Requirements are the primary focus in the systems engineering process because the process's primary purpose is to transform the requirements into designs.

Requirements analysis involves defining customer needs and objectives in the context of planned customer use, environments, and identified system characteristics to determine requirements for system functions [10].

\subsection{Studies of Livestock Information Systems}

A number of mobile phone information systems (IS) in the area of agriculture were studied. Agriculture includes, but not limited to, livestock, aquaculture, crop production, and forestry. Below are some of the IS that were studied.

\subsubsection{ICOW}

iCow is an Agricultural Information Service with a variety of products available as a subscription service through $* 285 \#$ to help farmers enhance productivity.

The app is a mobile phone cow calendar, which prompts farmers in Kenya on vital days of cows' gestation period. Helps them find the nearest Veterinary and Artificial Insemination providers. In addition, it collects and stores farmers' milk and breeding records, and it sends farmers best dairy practices.

\subsubsection{Kilimo Salama}

It is a mobile phone service that enables smallholder farmers to insure their agricultural inputs against adverse weather conditions.

\subsubsection{Kuza Doctor}

They call it 'A farmer's mobile toolkit from farm to fork'. It provides knowledge to farmers using SMS.

\subsubsection{SALI}

Sustainable Agricultural Livelihood Innovation (SALI) done in Mbeere, Embu by Christian Aid. It uses mobile phone technology to notify farmers of weather updates.

\subsubsection{Sangonet}

Sangonet is involved with an application that allows smallscale dairy farmers in East Africa to record the lactation history of their cows.

\subsubsection{Esoko}

Esoko is an information system that gives farmers, traders, agribusiness, and development projects tools to collect and share market information via SMS.

\subsubsection{Livestock Information Network and Knowledge System (LINKS)}

LINKS is a Livestock Information Network and Knowledge System which provides regular livestock prices and volume information on most of the major livestock markets in Ethiopia, Kenya and Tanzania. It provides market information that is available on request via SMS.

\subsection{Mobile Phone Technology in Tanzania}

The rapid expansion of mobile phone usage in Tanzania has been triggered by a highly competitive market and service diversification, with the operators now providing different mobile phone services such as voice and message transmission, data services, paging as well as Internet services. In terms of market shares, different companies, namely Vodacom and Zain (former Celtel) Zain/Airtel, tiGO and others are faring quite well [11].

The completion of the National ICT Broadband Backbone (NICTBB) and the booming number of mobile phone users has attracted and increased the number of Internet users in Tanzania to nine million [12]. However, Tanzania has a population of forty five (45) million people.

Historically, Internet services in Tanzania started in 1995. Five years later, in 2000 , the country had only 115,000 people connected to the Internet. Since then the number has kept growing significantly -reaching 9.3 million users in 2014; equivalent to 20 per cent of the 45 million people in Tanzania [12].

\section{Methodology}

The study used qualitative methodology. It is a research method used extensively by scientists and researchers studying human behavior and habits. Qualitative techniques are extremely useful when a subject is too complex to be answered by a simple yes or no hypothesis. These types of designs are much easier to plan and carry out. They are also useful when budgetary decisions have to be taken into account. Qualitative research methods are not as dependent upon sample sizes as quantitative methods.

\subsection{Research Methods}

The study used primary data, and secondary data. The primary data were collected through surveys, questionnaires, interviews, and direct observations. Secondary data were collected through books, articles, journals, and Internet searching.

Open Data Kit (ODK) tool was used to collect responses from the respondents, and geographical position. 


\subsection{Description of the Study Area}

Arumeru district is found in Arusha region, in the northern zone of Tanzania. Researchers collected data from fifteen wards, namely Poli, Sing'isi seela, Makiba, Maroroni, Mbuguni, Akheri, Kikatiti, Kikwe, King'ori, Maji ya chai, Nkoanrua, Nkoaranga, Nkoarisambu, Songoro, and Usa river.

\subsection{Research Design}

The research design refers to the overall strategy that you choose to integrate. The different components of the study in a coherent and logical way, thereby, ensuring you will effectively address the research problem; it constitutes the blueprint for the collection, measurement, and analysis of data [13].

This study used a case study research design. According to [14], a case study is an in-depth study of a particular research problem rather than a sweeping statistical survey or comprehensive comparative inquiry. We used Arumeru District as a case study.

\subsection{Sampling Design}

Sampling design covers all aspects of how the samples are specified and selected. The study used Simple Random Sampling without replacement, which is under the Probability Sampling, in a finite population. Under this sampling design, every item of the universe has an equal chance of inclusion in the sample.

In selecting sample design a researcher must pay attention to the following points: type of universe, sampling unit, source list, size of sample, parameters of interest, budgetary constraint, and sampling procedure [15]. We have considered these points in selecting the sampling design.

Advantages of simple random sampling include; bias is generally eliminated, and sampling error can be estimated.

\subsection{Inclusion/Exclusion Criteria}

Inclusion criteria are characteristics that the prospective subjects must have if they are to be included in the study, while exclusion criteria are those characteristics that disqualify prospective subjects from inclusion in the study. Inclusion and exclusion criteria may include factors such as age, sex, education, and profession.

This study used inclusion criteria whereby prospective subjects were supposed to be livestock keepers, who lived in Arumeru district.

\subsection{Population}

As of the 2012 census, Arumeru district has a population of 368,144 people. Livestock keepers are 102,134.

\subsection{Sample Size}

Sample size can be determined using different methods such as; sample size from a similar study, sample size table, and sample size calculator.

According to [16], using a population of 102,134 subjects, margin of error 5\%, confidence level 95\%, response distribution $50 \%$, the sample size is 383 .

In terms of the numbers we selected above, the sample size $\mathrm{n}$ and margin of error $\mathrm{E}$ are given by

$$
\begin{gathered}
\mathrm{x}=\mathrm{Z}(\mathrm{c} / 100) 2 \mathrm{r}(100-\mathrm{r}) \\
\mathrm{n}=\mathrm{N} \mathrm{x} /((\mathrm{N}-1) \mathrm{E} 2+\mathrm{x}) \\
\mathrm{E}=\operatorname{Sqrt}[(\mathrm{N}-\mathrm{n}) \mathrm{x} / \mathrm{n}(\mathrm{N}-1)]
\end{gathered}
$$

where $\mathrm{N}$ is the population size, $\mathrm{r}$ is the fraction of responses that you are interested in, and $Z(c / 100)$ is the critical value for the confidence level c.

A total of 210 smallholder farmers and 30 livestock experts were sampled to represent the population. The livestock experts included fifteen (15) field livestock officers, one from each ward. Ten (10) District livestock officers, and five (5) livestock researchers from Nelson Mandela African Institution of Science and Technology (NM-AIST), Livestock Training Agency (LITA), and National Artificial Insemination Center (NAIC).

Similar studies were conducted by [17], [18]. They used similar sample sizes.

\subsection{Analysis of Data}

Data analysis was conducted using Statistical Package for Social Sciences (SPSS). Data were previously collected using Open Data Kit (ODK) tool and exported to SPSS.

\subsection{Case Study Area Mapping}

Open Data Kit (ODK) tool helped in collecting data and geographical position of the respondents during requirements gathering. Positions were recorded, exported to KML file format, and plotted using Google Earth, see Figure 1 below. Geographical positions of the respondents show importance of using mobile phones for exchange of information.

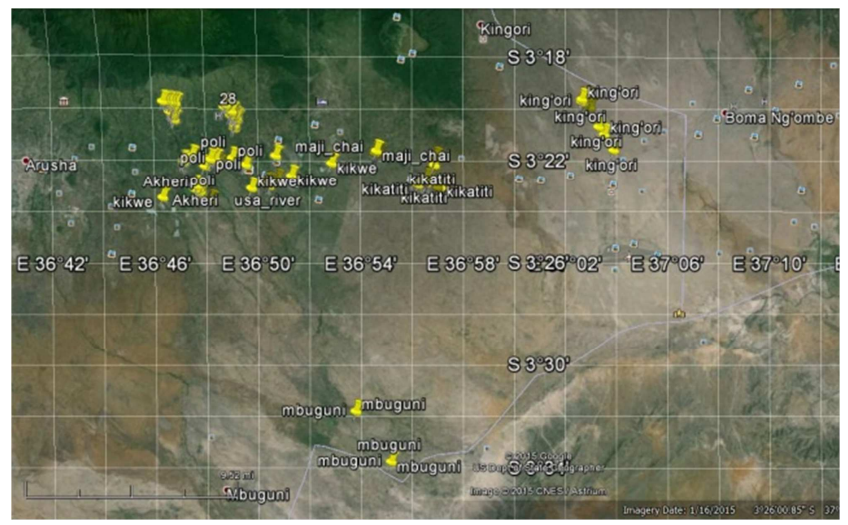

Figure 1. Distribution of the respondents in the research area.

Figure 2 below shows the frequency distribution of respondents in fifteen wards that are located in Arumeru district, Arusha region. 


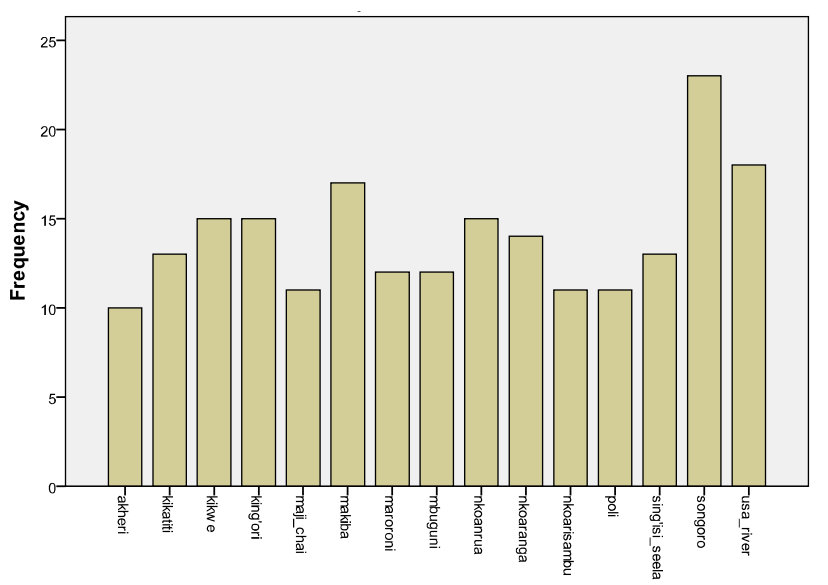

Figure 2. Frequency distribution of the respondents in the wards.

Among the livestock keepers who were interviewed, males were $103(49 \%)$, while females were 107 (51\%). Age distribution was $2(1 \%)$ below 20 years, between 20 and 39 were $68(32.4 \%)$, between 40 and 55 were 103 (49\%), and above 55 were $37(17.6 \%)$.

\section{Results and Discussion}

This section shows results and discussion of the study basing on the challenges facing field livestock officers, types of information shared, how they share information, how often livestock keepers communicate with livestock field officers, how they record data, access to marketing information, mobile phone usage, advantages of a proposed framework, system requirements, quantifiable benefits, and requirements model.

\subsection{Challenges Facing Field Livestock Officers}

Field livestock officers face a number of challenges, which impair their routine activities. These include; they serve a large number of livestock keepers who are far and scattered; lacking means of transport; lack of enough funds to support extension services; difficulties in accessing published research findings;

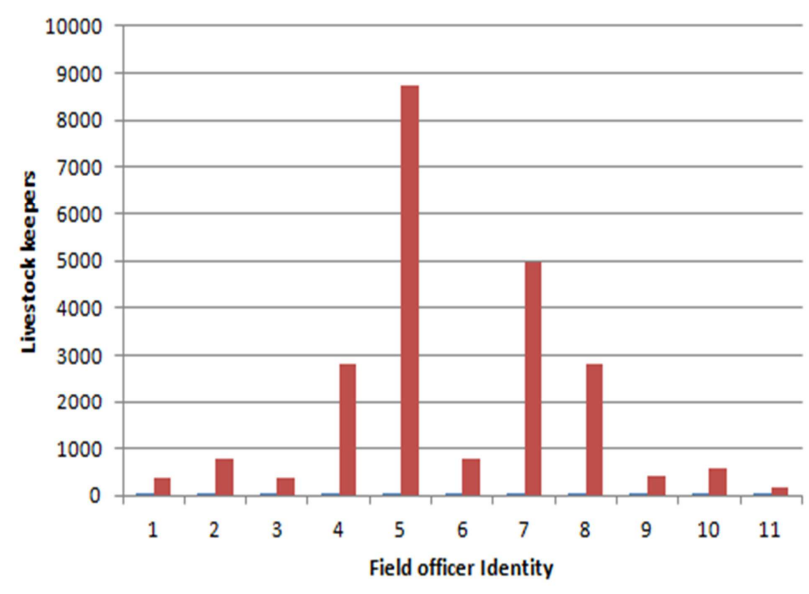

Figure 3. Number of livestock keepers per livestock field officer.
On average each field officer serves 2078 livestock keepers. However, the ideal ratio is one livestock field officer to seven hundred (700) livestock keepers. This increases challenges to the field officers to serve livestock keepers.

\subsection{Types of Information Shared}

User requirements gathering process was conducted among the livestock keepers and livestock experts. The livestock keepers mentioned market information, disease outbreaks, vaccinations and treatments, weather, and modern livestock husbandry. On the other hand, livestock experts mentioned market information, disease information, and weather.

\subsection{How They Share Information}

The study shows that, smallholder livestock keepers mainly share information through the field livestock keepers. They use face to face mechanism. This is a challenge as livestock keepers are geographically dispersed, and the field livestock officers do not have reliable means of transport. Another way that they use is to make a phone call. However, solving the livestock problems on a phone is also another challenge.

Sometimes the smallholder livestock keepers share information through neighbors, and mass media such as radio and television.

\subsection{How Often They Communicate with Livestock Field Officers}

Analysis shows that majority of livestock keepers 116 (55.2\%), communicate with the livestock field officers when problems arise, followed by 46 (21.9\%), who communicate monthly. Others communicate either weekly, or do not communicate at all.

Livestock keepers communicate with livestock field officers when the problems arise because the livestock field officers do not follow the regular visit timetable. Some livestock keepers do not know their field officers, and some do not even know if they exist.

\subsection{How They Record Data.}

In most of the farms, data is recorded on hard copy and then it is posted to herd registers for compilation, recoding and feeding in computer for analysis. This is often not a regular process even in many institutional farms. The study observed the same trend in Arumeru district; however some livestock keepers kept their data by mere memorizing.

\subsection{Access to Marketing Information}

Livestock keepers need market information to sell their products and by products, or buy the commodities. Study found that, they access market information through face to face, mobile phones, and visit to markets. There is no livestock information system which is necessary for exchange of information. 


\subsection{Mobile Phone Usage}

Among the livestock keepers 206 (98.1\%) own phones. Only $4(1.9 \%)$ do not own phones. However, only 11 respondents own smart phones. They use their mobile phones for Short Message Services (SMS), making and receiving calls, and mobile money transfer.

They support the idea of using mobile phones for exchange of information among the livestock stakeholders.

\subsection{User Requirements}

The study came up with the following requirements; receive prices of their products and byproducts, buyer information, livestock auctions, feed and fodders, insecticides, pesticides, and communication with livestock field officers, milk production, milk sales, disease outbreaks, Vaccinations, Births, Deaths, and slaughter.

\subsection{Quantifiable Benefits}

Below are some of the quantifiable benefits that smallholder livestock keepers will receive;

(1) Less time wasted in search for livestock information.

(2) Better prices for their products and by-products.

(3) Increasing profit.

(4) Reduced product and by-product losses.

(5) Fewer journeys in search for information.

(6) More volume production.

(7) Better livestock keeping practices.

\subsection{Requirements Model}

Requirements model captures functional requirements from user perspective. It makes use of Use Case diagrams. It has inputs and outputs. Inputs are system requirements specifications, documentations of existing systems, and practices that are to be followed, exchanges between developers and users and specifiers. Outputs are use case model, and concise descriptions of use case.

\subsubsection{Existing Data Flow Diagram}

The existing model puts the livestock field officer at the middle between the livestock keeper and other actors. Every information passes through the livestock field officer.

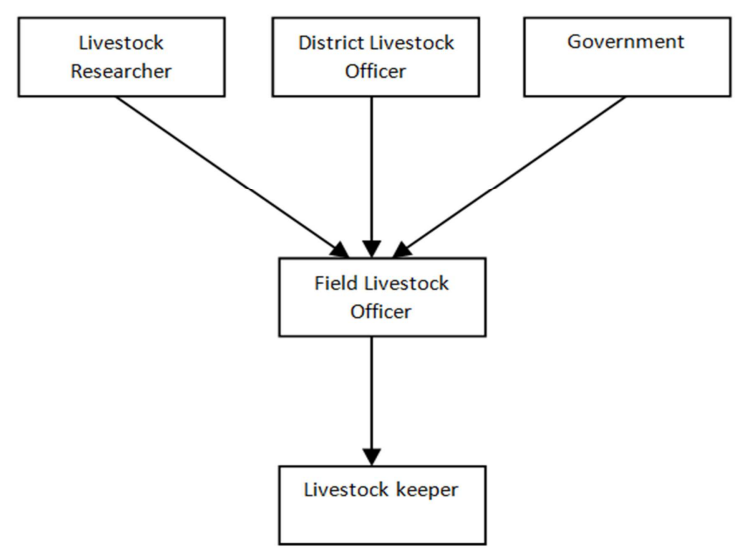

Figure 4. Existing data flow model.
Field livestock officers face challenges in dispersing useful information to livestock keepers, who are geographically scattered. The field livestock keepers do not have reliable means of transport and communication. Therefore there are barriers in conveying modern livestock practices and other extension services.

\subsubsection{Use Case Model}

Use case model is a collection of use cases.

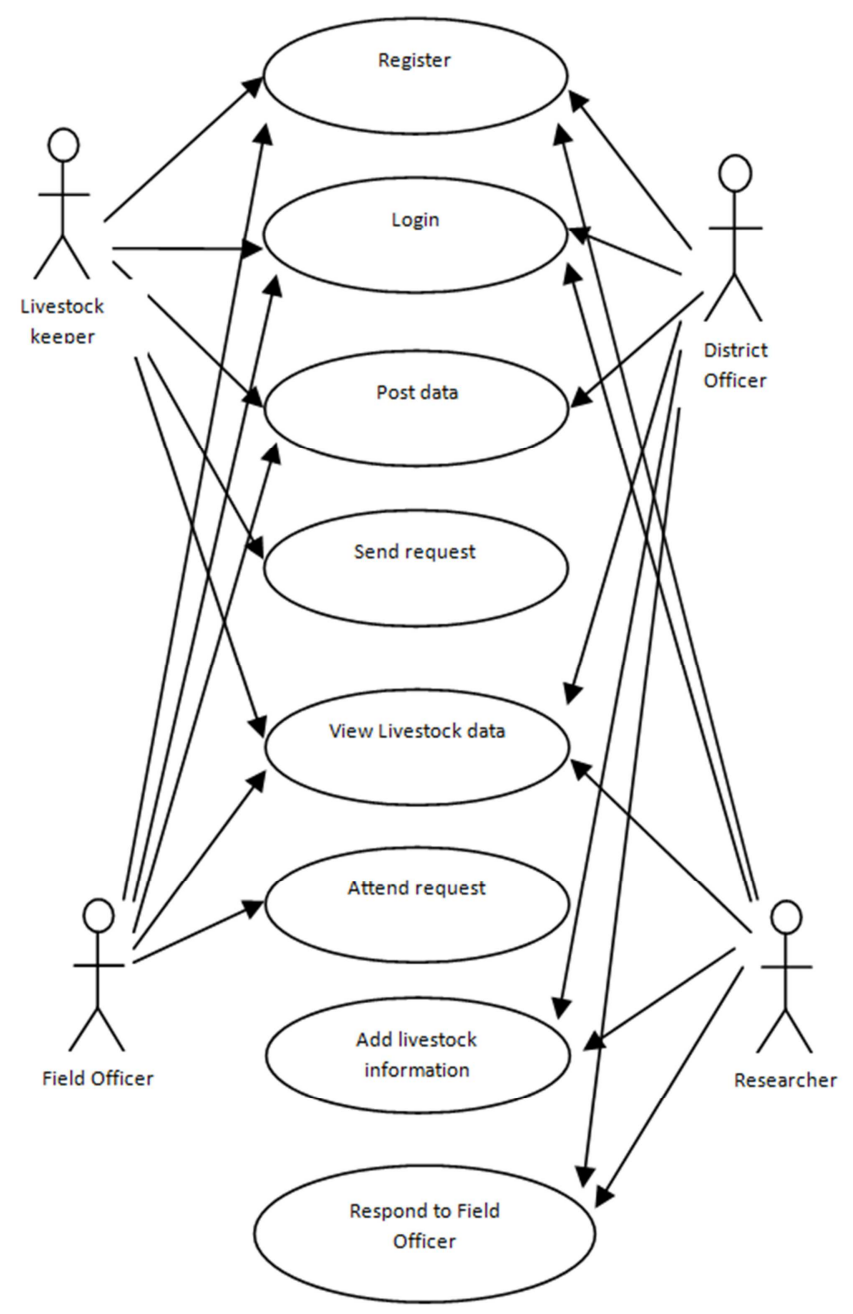

Figure 5. Use case model.

The model has four actors who are livestock keeper, livestock field officer, livestock researcher, and livestock district officer.

Livestock keeper wants to register in the system, login using his/ her username and password, post data, send request or query to the livestock field officer, and view livestock information on various aspects such as feed and fodder, disease outbreak, pesticides, and market prices.

Livestock field officer, apart from registering and logging in the system, he/ she needs to receive livestock data from livestock keepers, post data, view livestock data from livestock keepers, livestock district officers, and livestock researchers.

Livestock researcher registers and logs in to the system. 
$\mathrm{He} /$ she needs to view livestock data from livestock keepers and do data analysis so as to come up with scientific solutions to the problems.

Livestock district officer supervises the livestock field officers, view livestock data from the livestock keepers, view research findings from researchers, and can respond to livestock field officers.

\subsection{Advantages of a Proposed Framework}

Findings show that, many frameworks were addressing issues of crop production, and their value chain. The emphasis was in the marketing of crops. In addition, the apps are using SMS to receive information. The study did not find the frameworks which address difficulties and challenges of smallholder livestock keepers.

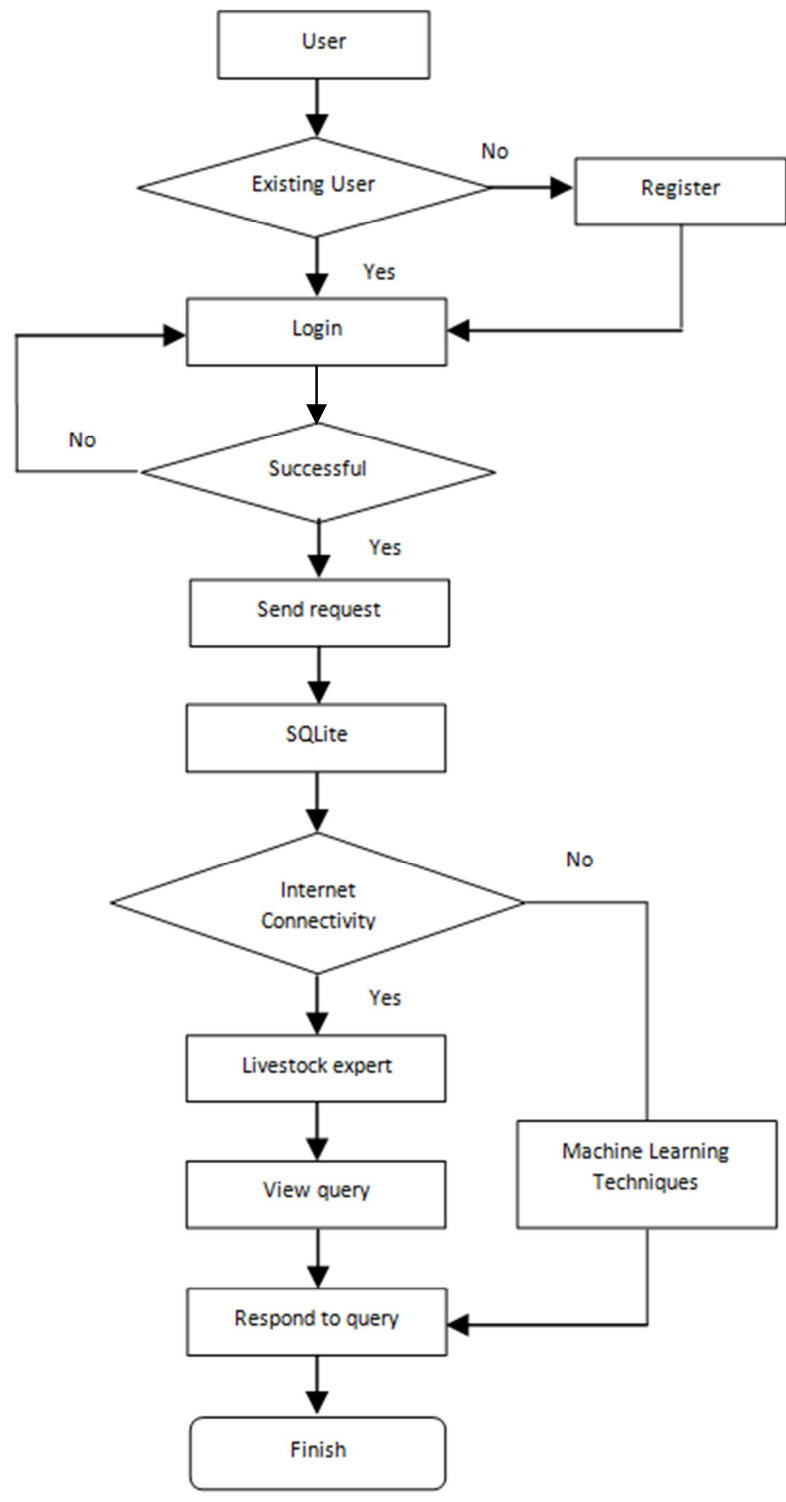

Figure 6. Data flow diagram.

Smallholder livestock keepers need the framework that will enable them receive prices of their products and byproducts, buyer information, livestock auctions, diseases, feed and fodders, insecticides, pesticides, and communication with livestock field officers.

The existing mobile phone solutions fail to work in poor connectivity areas. They only work where there is constant connectivity. They do not use machine learning techniques to respond to the queries. They are not able to make predictions that can assist operations of livestock information systems.

The proposed framework will enable operations of livestock information systems in poor connectivity areas through making use of machine learning techniques. It will be capable of responding to queries from livestock keepers without assistance from the livestock field officers.

\section{Conclusion}

Mobile application based solutions, which are currently widely proposed to facilitate the process, fail to perform in poor connectivity areas, and do not address issues that smallholder livestock keepers face. This study does the requirements analysis and comes up with requirements model. It paves a way for a machine learning based framework which will enhance the performance of mobile application based solutions in poor connectivity areas.

Results show that smallholder livestock keepers are geographically scattered and depend on the field livestock officers for exchange of information. Their means of communication are mainly face to face, and mobile phones. They do not use any Livestock Information System. The proposed framework will enable operations of Livestock Information System in poor connectivity area, where majority of smallholder livestock keepers live.

\section{Future Work}

In the future we plan to design Machine learning based framework for enhancing performance of livestock mobile application system. We will use that design to develop and implement machine learning - based application framework for enhancing performance of livestock mobile application systems

\section{Acknowledgement}

Appreciations are expressed to the Nelson Mandela African Institution of Science and Technology (NM-AIST), Livestock Training Agency (LITA-Tengeru), National Artificial Insemination Agency (NAIC), and Arumeru District Council, for making this study a success.

We would like to thank the anonymous reviewers for their valuable feedback.

\section{References}

[1] C. Rolland et al., "A proposal for a Scenario Classification Framework", Journal of Requirements Engineering, Vol. 3 No. 1, 1998, Springer Verlag, pp. 23-47. 
[2] Schneider, G. and J. Winters, "Applying Use Cases - A Practical Guide", Object Technology Series, ed. J. Booch, Rumbaugh. 1998: Addison Wesley.

[3] Leffingwell, D. and Widrig, D. (2003) "Managing Software Requirements". 2nd Edition, Addison-Wesley, Boston, MA, USA.

[4] Ministry of Livestock and Fisheries Development (2010), "Livestock Sector Development Strategy", Tanzania.

[5] Robert J. Mcqueen (1994), “Applying Machine Learning to Agricultural Data", Management Systems, University of Waikato, Hamilton, New Zealand.

[6] Joris Vanderschrick (2011), "System Requirements Analysis: The first step to value-based system development", Verhaert Embedded Systems Development, Belgium.

[7] Jaelson Castro and John Mylopoulos (2002), "Information Systems Analysis and Design",

[8] S. Pfleeger and J. Atlee (2006), "Software Engineering Theory and Practice", Third Edition, Prentice Hall.

[9] Rai, A., Dubey, V., Chatuverdi, K. K., Malhotra, P. K., (2008). "Design and development of data mart for animal resources", Journal of Computers and Electronics in Agriculture. 64 (2), p111-119.

[10] Defense Acquisition University (2001), "Systems Engineering Fundamentals", Defense Acquisition University Press, Fort Belvoir, Virginia.
[11] Bhavnani, A A., Won-Wai Ciu, R., Janakiram, S. and Silarszky, P. (2008), "The Role of Mobile Phones in Sustainable Rural Poverty Reduction", Report for the World Bank ICT Policy Division, Global Information and Communications Department, Washington DC.

[12] The Citizen, 2014, “Tanzania's Internet users hit 9m", published online, Wednesday October 1, 2014.

[13] De Vaus, D. A. (2001), "Research Design in Social Research", London: SAGE.

[14] Yin, R. K. (2003), "Case Study Research: Design and Theory", Applied Social Research Methods Series. CA: Thousand Oaks.

[15] Kothari, C. R. (2006), "Research Methodology, Methods and Techniques", New Delhi, India: New Age International Publishers.

[16] Calculator, R. (2014, December 17), "Raosoft", Retrieved December 17, 2014, from http://www.raosoft.com/samplesize.html:

[17] Bernard Mussa, Zaipuna Yonah, Charles Tarimo, (2014), "Towards a Mobile - Based DSS for Smallholder Livestock Keepers: Tanzania as a Case Study", International Journal of Computer Science and Information Security, Vol. 12, No. 8, August 2014.

[18] Gladness Mwanga, Zaipuna Yonah, (2014), "ICT as a tool for improving information flow among livestock stakeholders: a case study of Tanzania", International Journal of Computer Science and Information Security, Vol. 12, No. 8, August 2014. 